\title{
EXPLICACIONES, MECANISMOS Y REACCIONES QUÍMICAS
}

\author{
JUAN BAUTista BENGOETXEA \\ Oliver TODT
}

\begin{abstract}
In this paper we propose an enhanced notion of mechanistic explanation that is in accordance with chemical practice. From the necessity of understanding practices of explanatory inquiry in organic chemistry from a dynamic point of view in which intervention and contrafactual experimental settings are emphasized, a non-nomological-deductive mechanistic explanation view is presented. Not sufficiently emphasizing the explanatory and dynamic features has led some philosophers of chemistry to doubt about this view's validity in this field of science. In this article we claim that a well articulated consideration of those features in the mechanistic explanation view may both strengthen this model and correctly account for the explanatory activities in the case of the study of organic chemical reactions.
\end{abstract}

Keywords: Explanation; mechanism; organic chemistry; experimental practices.

\section{Introducción}

La explicación científica constituye uno de los temas más candentes en la filosofía de la ciencia desde que varios autores en la década de 1990 recogieran el testigo que previamente Hempel y Oppenheim (1948), Nagel (1961) y Hempel (1965) trajeran consigo a la disciplina (Cfr. Psillos 2002, p.215-7). Los desarrollos al respecto de los últimos 25 años han elaborado aun más la noción con detalles novedosos vinculados a las tareas que la actividad científica real lleva a cabo de maneras cada vez más complejas. Han sido de gran ayuda para el estudio de la explicación los nuevos enfoques de la filosofía de la ciencia a partir de 1980, en particular dos: el nuevo experimentalismo y la filosofía de las prácticas científicas. ${ }^{1}$ Cada uno de ellos ha originado imágenes de la ciencia complementarias a muchos resultados de los estudios más tradicionales 'volcados en la estructura lógico-lingüística de las teorías' que concebían la ciencia sólo como conocimiento proposicional. Más allá de algunos estudios previos sobre las prácticas científicas (Fleck 1935, Polanyi 1958), sólo posteriormente han emergido los nuevos enfoques que han analizado la experimentación y las prácticas.

En particular, aquí nos interesa hacer hincapié en la elaboración de mecanismos y de explicaciones en la ciencia experimental de laboratorio. La química, más allá de su indispensable faceta teórica (modelos, simulaciones, cálculos computacionales), depende en un alto grado del trabajo en laboratorios (experimentación) y de muchos

Principia 18(3): 393-417 (2014).

Published by NEL — Epistemology and Logic Research Group, Federal University of Santa Catarina (UFSC), Brazil. 
tipos de tecnologías, desde la instrumentación en su sentido más clásico a las tecnologías de la información. Al ser, además, un ámbito en el que la noción clásica de explicación — por cobertura legal del modelo nomológico-deductivo (ND)— encuentra problemas serios a los que se pueden contraponer alternativas diversas (Salmon 1989; Kitcher y Salmon 1989; Psillos 2002; Lipton 2004), proponemos un intento de abordar el ámbito de la química orgánica y sintética desde un enfoque filosófico de las prácticas experimentales con el objetivo de elaborar una noción de explicación más acorde a ese tipo de actividad. Para ello, proponemos la explicación basada en mecanismos ('EBM' en adelante) como marco filosófico para nuestro análisis de las prácticas de indagación explicativa en química orgánica. Debido a la carencia de reflejo de los aspectos dinámicos y diacrónicos de los procesos de modelación de los mecanismos de fenómenos de reacción, el marco ha sido criticado por algunos filósofos de la química (Tabery 2004; Ramsey 2008).

Aquí consideramos que la EBM es un enfoque salvable, si bien mejorable. En un intento por aportar a su mejora, analizamos varios procedimientos de examen químico de producción de fenómenos con el fin de incorporar aspectos dinámicos que mejoren los resultados epistemológicos del estudio de la explicación acorde a las prácticas reales en química orgánica. Como consecuencia de ello, vemos que en química orgánica, sus prácticas de análisis de los mecanismos de reacción se pueden concebir como actividades de generación de modelos (posibles, plausibles y reales) de mecanismos que tratan de dar cuenta del proceder diacrónico de las reacciones químicas orgánicas. Son prácticas con un carácter intervencionista visible en las modificaciones que se realizan normalmente en las condiciones de reacción o en los propios reactivos, bajo un patrón contrafáctico, y que permiten derivar la pertinencia de unos mecanismos y la irrelevancia de otros. Convenimos que esto se refleja adecuadamente en un enfoque EBM, si bien bajo la advertencia de no desestimar al menos los aspectos novedosos considerados. Con ello pretendemos establecer un primer paso de un procedimiento que capture más satisfactoriamente la forma y el contenido de la actividad explicativa científica en general, y de la química en particular.

El artículo se estructura como sigue. En la primera sección se defiende la necesidad de comprender la actividad científica desde una perspectiva dinámica, en la que la intervención se sume a la observación, y la explicación no nomológicadeductiva sustituya los enfoques que requieren leyes para caracterizar el quehacer científico en química. En la sección segunda exponemos el enfoque más importante de la explicación basada en mecanismos, a partir del cual presentamos la idea básica de mecanismo en ciencia y las nociones complementarias de modelos. La sección tercera conforma el núcleo del escrito y en ella defendemos qué elementos, además de los que el enfoque de mecanismos propone, hacen que un mecanismo sirva de fundamento para una explicación propiamente química, en particular para una ex- 
plicación de una reacción química. En la sección cuarta presentamos las conclusiones más importantes del artículo.

\section{Experimentación, fenómenos y explicaciones en ciencia}

Aunque no el único ni necesario, un eje fundamental sobre el que pivotan muchas de las ciencias naturales es la experimentación. La actividad experimental consiste en algo más que la realización de un experimento y la observación de un resultado. Se trata de una actividad compleja que incorpora también el diseño y la fabricación de instrumentos, su calibración, la verificación de su funcionamiento correcto y su mantenimiento. Pero la producción, estabilización y aceptación de un fenómeno 'natural' - aunque sea creado en el laboratorio o sintetizado- implica operaciones de diversa índole y gran parte de conocimiento tácito que no se transfiere fácilmente. Hay que tomar en consideración el saber-cómo respecto al diseño, a la construcción y al manejo de tecnologías muy específicas, y a los procedimientos y habilidades para reconocer cuándo un experimento funciona correctamente y cuándo no. Todo ello suma el hacer a la observación en ciencia.

Esta tarea 'hacedora' (cfr. Galison 1996) en realidad consiste en que los científicos experimentadores creen fenómenos en el laboratorio y no 'simplemente' los descubran (Hacking 1983, p.230). La explicación de gran parte de los fenómenos científicos, por ende, parte de un proceso previo de creación (Hacking 1983, 1989) que la carencia de fenómenos directamente accesibles en la naturaleza fuerza. Si bien no todos, muchos de los denominados 'fenómenos naturales' del conocimiento científico estable son dependientes de idealizaciones y han sido producidos en el laboratorio (Hacking 1983, p.224; Chang 2004, p.166). ${ }^{2}$ Pues bien, la explicación científica no puede desestimar este vínculo de cuasi-necesidad entre ciencia e intervención experimental consistente en crear artificialmente, producir, refinar y estabilizar fenómenos (Hacking 1983, p.230) hasta hacer que un experimento sea exitoso (Hacking 1983, p.229).

El diseño de las condiciones que se establecen en la experimentación y de los fenómenos que se pretenden estudiar en pocas ocasiones se da de forma natural; es decir, "en ausencia del aparato" (Harré 2004, p.180; 2009, p.7). ${ }^{3}$ Esto es bastante habitual en química, cuyo carácter creador supera al puramente analítico —no olvidemos que la principal actividad de la disciplina hoy es la síntesis (Chamizo 2013, p.631). ${ }^{4} \mathrm{El}$ 'elemento creador' que Hacking enfatiza podría tomar la forma de síntesis en química, la forma de reacciones químicas que generan nuevos productos, su aislamiento y su procesamiento posterior, así como la investigación de sus propiedades. Por lo tanto, el experimento químico crea, en un aparato o dispositivo, un fenómeno (una sustancia) empleando solventes como instrumentos (Chamizo 2013, 
p.636). Una forma de visibilizar y explicar este proceso creador es el suministro de un mecanismo de reacción, como veremos en las secciones 3.2 y 3.3.

\subsection{Naturaleza Explicativa Química}

La hipótesis de la ciencia creadora, no sólo observadora y representacional, no debe dejar de lado los aspectos teóricos de la modelación química. Éstos se vierten en forma de estructuras de varios tipos con la finalidad de aprender acerca de sistemas reales o fenómenos creados en el laboratorio e intervenir en ellos (Woodward 2002, p.97-98). Esto, aunque indiscutible, no está exento de cierta tensión inherente a la relación entre precisión y generalidad (Weisberg 2013, p.109-10), muy delicada en el ámbito químico. Por ejemplo, los modelos de la química cuántica -los espacios de estados- normalmente correlacionan distancias de partículas sub-atómicas con la energía total de los sistemas químicos, pero hay otros modelos menos precisos, aunque más útiles para otros propósitos, que procuran responder a demandas químicas de teorías más fenomenológicas (reflejo de los aspectos dinámicos de las reacciones, modelación de la estructura molecular y la simetría mediante grafos y grupos, etc.). La tensión precisión-generalidad, no obstante, no desaparece del todo, sino que se traslada hacia distintos ámbitos de la propia química, habitualmente los que diferencian campos teóricos próximos a la física de los campos más propiamente químicos que emplean conceptos, modelos y teorías cercanas a la labor experimental en el laboratorio. ${ }^{5}$

Muchos químicos experimentales no comparten el interés por calcular con precisión meridiana o por recurrir a teorías de alto nivel (Berson 2008, p.148). Su apuesta es la 'moderación': por un lado, consideran que los modelos simples desde la perspectiva química son necesarios para la teorización química (Hoffmann 1995, 1998; Carroll 1998; Hendry 2001), si bien, por otro lado, reconocen que hay que elegir modelos suficientemente precisos para los propósitos de computación, inevitables hoy en día en química. Solo que estos modelos a su vez deben ser lo bastante simples para que comprendamos lo que describen. Si no es así, los modelos se convierten en cajas negras y no entendemos su 'quehacer' (cfr. Carroll 1998, Sec. 1.4; Hoffmann 1998, p.2).

El enfoque explicativo ND incorporaría esta exigencia de comprensión sólo parcialmente (Cfr. Scerri 1991, p.322). Hoffmann (1998, p.2-3) lo asocia al reduccionismo de los empiristas lógicos ${ }^{6}$ y le contrapone un procedimiento explicativo basado en el análisis del fenómeno en términos únicamente de su propia disciplina e indagando en las relaciones que mantiene con otros conceptos de igual complejidad. Hoffmann critica el reduccionismo radical y defiende la existencia de conceptos irreducibles de la química respecto de los de la física, caso de la aromaticidad, la interacción esteárica, la acidez o la basicidad, la noción de grupo funcional o la de efecto sustituyente 
(Theobald 1976, p.209; Hoffmann 1998, p.3). Se trata de constructos que no se pueden matematizar completamente y que la explicación de su papel en mecanismos de reacción depende también de procedimientos empíricos (experimentales).

Los enfoques filosóficos más atentos a la experimentación reclaman, por lo tanto, factores fenomenológicos de reacción química (electrostática [cargas, dipolos], interacciones donante-aceptor, orbitales, etc.) a la hora de proporcionar explicaciones, y aunque no exigen un giro radical y desestimar la teoría física por completo, reconocen que la explicación-comprensión en química es algo más que el modelo ND. Sin obviar la importancia que éste tuvo en su momento (Woodward 2003, p.190-1), parece conveniente que aparezcan en escena concepciones filosóficas de la explicación que respondan al esbozo que Hoffmann promociona cuando escribe sobre ese otro modo de explicación.

\section{Explicación y Mecanismos}

Si bien la explicación científica ha sido una de las cuestiones más debatidas y expuestas a crítica en la filosofía de la ciencia del siglo XX, ${ }^{7}$ la explicación mecanística ha estado ausente casi en su totalidad de los análisis filosóficos. Machamer, Darden y Craver (2000) publicaron un artículo seminal que abrió paso a una línea de investigación realmente productiva e interesante. ${ }^{8}$ Motivos para su viraje se encuentran ante todo en los estudios filosóficos de ciencias que no son la física. Se observa que en las ciencias de la vida la explicación no recurre apenas a leyes, sino que consiste en la descripción de mecanismos propiamente dichos. ${ }^{9}$ En biología molecular, por ejemplo, se han propuesto diversos mecanismos que explican los fenómenos de la fermentación y la síntesis proteínica, entre otros (cfr. Bechtel 2011). En general, se puede afirmar que las neurociencias y las ciencias de la vida desarrollan y manejan EBMs a la manera clásica mencionada. En el caso de las ciencias biológicas, cabe resaltar la importancia de las explicaciones funcionales-teleológicas. Tal y como Nunes-Neto y El-Hani (2009, p.358-9) destacan, el análisis funcional de Robert Cummins concede a la noción de función un rol mediante el cual, en vez de buscar causas históricas con las que explicar diacrónicamente la existencia de algún estado real de cosas, en realidad proporciona sincrónicamente explicaciones de las funciones de los componentes de un sistema complejo a partir del análisis de alguna capacidad que el sistema presenta como un todo. Estas capacidades sistémicas, continúan Nunes-Neto y El-Hani, se explican por medio de un análisis en términos de las capacidades de las partes componentes, a saber: las funciones a las que Cummins se refiere.

\subsection{Estructura de los Mecanismos}

Un mecanismo se caracteriza como el complejo compuesto de "entidades y actividades organizadas de modo que producen cambios regulares desde las condiciones de 
inicio a las condiciones finales" (Machamer et al. 2000, p.3). Son las entidades y actividades que causan un fenómeno. El mecanismo es lo que constituye un fenómeno. En particular, un mecanismo causal es una secuencia de acontecimientos "desde un estado inicial a un estado final, conectados mediante bifurcaciones de procesos causales, conjuntivas e interactivas" (Forge 1998, p.78; Machamer 2004, p.27-8). ${ }^{10}$ Sus entidades son las partes activas de la producción de un fenómeno determinado, así como los componentes estructurales del mecanismo. Las operaciones, por su parte, son los procesos o cambios que involucran a las partes (Machamer 2004, p.29; Bechtel 2007, p.14).

Darden propone cuatro características principales para caracterizar un mecanismo (Darden 2006, p.279-80): los componentes (entidades, actividades, módulos), la ordenación espacial de éstos (localización del mecanismo, estructura, orientación espacial, conectividad con otros objetos de ese espacio y compartimentación) (Bechtel y Richardson 2010, especialmente su capítulo 2, "Complex Systems and Mechanistic Explanations"), sus aspectos temporales (orden, proporción, duración y frecuencia) y las localizaciones contextuales (estar situado en una jerarquía de fenómenos (constitutiva) y en una serie causal (etiológica)). El análisis del mecanismo se desarrolla por medio de procesos de descomposición y localización: el primero consiste en el análisis de un fenómeno en sus actividades que, cuando se organizan apropiadamente, exhiben el fenómeno en cuestión. La localización es la identificación de las actividades y los componentes (Craver y Bechtel 2006, p.473).

Un mecanismo se puede concebir como un conjunto organizado de las partes (y sus operaciones) correlacionadas que causan un fenómeno específico. La descomposición delimita un fenómeno en una serie organizada de actividades. Más específicamente, es el proceso mediante el cual los científicos disciernen niveles de mecanismos, organizando las partes y las operaciones en referencia con el fenómeno investigado (Craver 2002, p.S88-S89). La localización delimita un fenómeno en componentes que realizan una serie de actividades dentro de un espacio dado. El proceso de descomposición y localización se deriva a menudo a partir de una combinación de observación y resultados experimentales, hipótesis y manipulación del fenómeno con el fin de extraer indicios sobre cuáles son sus partes, qué hacen y cómo se organizan (Bechtel 2007, p.35). La descomposición normalmente comienza con una propuesta de qué componentes son los responsables del fenómeno, hipótesis que toman la forma de modelos de explicación (Craver y Bechtel 2006, p.474). ${ }^{11}$

El descubrimiento del mecanismo parte habitualmente de un fenómeno problemático (Darden 2006, p.289). La elección de dicho fenómeno y la manera de caracterizarlo delimitarán el modelo o hipótesis posible, o espacio de modelos, y dirigirán la construcción de una EBM para ese fenómeno (Craver y Darden 2001, p.123). Y aunque parezca arriesgado, el proceso práctico, real, de la elaboración del mecanismo procede habitualmente por 'inferencia de la mejor explicación': se generan modelos 
hipotéticos que procuran dar con el mecanismo o mecanismos subyacentes a un fenómeno y que apuntan al lugar donde se causa el fenómeno. Una vez se determina tal lugar, el subsistema que forma el mecanismo se localiza y descompone en sus diversas localizaciones, partes y funciones (Bechtel y Richardson 1993). Se construye así un modelo del mecanismo con el fin de representar el mecanismo real que causa el fenómeno.

\subsection{Modelos de Mecanismos}

Modelar es fundamental en ciencia, pero los modelos no sólo buscan explicar. Básicamente sirven para predecir con rigurosidad, para sintetizar datos, para diseñar experimentos y su forma es variable, desde la de los modelos lingüístico-naturales hasta la de los materiales (físicos y virtuales (computación)) o matemáticos, entre otros (Bogen 2005, p.405). Aquí nos interesan los modelos con la propiedad añadida de 'ser una explicación' (Craver 2006, p.355).

Entre los muchos sentidos diferentes de 'modelo' en ciencia y entre sus diversos fines científicos (Cfr. Giere 1999; Balzer y Moulines 1996, p.5; Galison 1996; Weisberg 2013, p.7-23), cabe destacar que los modelos también se emplean con el fin de representar y actuar sobre sistemas fenoménicos particulares. Pocos modelos son isomorfos con el fenómeno estudiado; la abstracción de muchos ingredientes del sistema modelado es práctica habitual. Esto significa que los modelos habitualmente son aproximados, pues el investigador emplea asunciones simplificadoras a la hora de procurar aplicar un formalismo particular.

Un modelo puede que sea útil y, aun así, que no sea una explicación. Para mostrarlo con claridad, Craver (2006, p.356) distingue tres pares de nociones que recogemos aquí: (i) modelos fenoménicos versus explicaciones, (ii) bosquejos versus explicaciones completas, y (iii) modelos posibles versus modelos reales. Si lo que pretendemos es identificar si un modelo empleado en ciencia es una explicación y no otra cosa distinta, estas distinciones son requisito mínimo para comenzar la tarea (cfr. Bengoetxea, Todt y Luján 2013).

(i) Subyace a nuestras consideraciones la idea de que un modelo que pretenda ser adecuado al fenómeno estudiado y apropiado explicativamente, será un modelo que dé cuenta de todos los aspectos pertinentes del fenómeno; si la cantidad de éstos es reducida, el modelo deja de ser explicativo. Puede que sea un modelo fenoménico excelente respecto de esos aspectos que modela, pero puede que a su vez no constituya una explicación. Los modelos simplemente fenoménicos no son, por lo tanto, explicaciones.

En ciencia interesan los modelos que sí son explicaciones. Hay motivos variados para creer que esto es así, pero uno que sobresale es el que enfatiza que si el modelo es una explicación, es más útil que un modelo meramente fenoménico en lo que res- 
pecta al menos a la manipulación, el control y la intervención (Hacking 1983; Craver y Darden 2013, p.120; Machamer 2004, p.28; Woodward 2002, p.95), tres tipos de acciones que son fundamentales en las ciencias experimentales. Por un lado, la explicación es asunto de mostrar patrones sistemáticos de dependencia contrafáctica (Woodward 2003, p.191). Dice no sólo cómo funciona de hecho un sistema examinado, sino también cómo lo haría en caso de manipulación o de intervención, en condiciones de variación del estado de cosas. Cuanto más amplio sea el abanico de estas intervenciones, más satisfactoria será la explicación (Craver y Darden, 2013, p.133-5). Por otro lado, la explicación añade algo a lo que un modelo fenoménico proporciona (responder a ciertas preguntas acerca de un conjunto limitado de aspectos del sistema estudiado), a saber: responde al porqué del tipo de relaciones que se dan en un modelo fenoménico y explicita las condiciones en las que dichas relaciones se verían forzadas a modificación (Craver 2006, p.358). En este sentido, las explicaciones son más útiles que los modelos fenoménicos en el sentido de que cubren un rango más amplio de contingencias posibles.

(ii) Ilustremos el continuo a lo largo del cual se pueden situar los modelos que describen mecanismos. Los polos opuestos son un bosquejo de mecanismo y una descripción idealmente completa del mecanismo. En medio, el posicionamiento puede variar de modo gradual (Fig. 1):

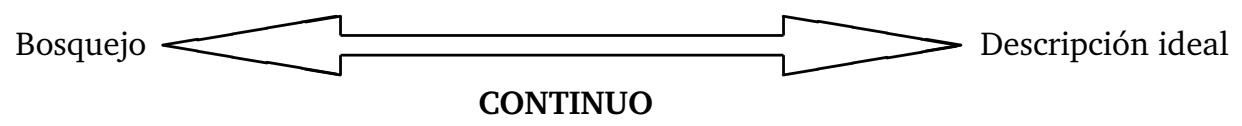

Figura 1: Modelos de mecanismo: (a) bosquejo, (b) descripción completa, (c) esquemas intermedios

Veamos cada elemento individualmente:

(a) Bosquejo: según Craver (2006, p.360), es el modelo más incompleto de un mecanismo. Caracteriza algunas partes, actividades y aspectos de la organización del mecanismo, pero exhibe vacíos y brechas en el seno del mecanismo (cfr. Craver y Darden 2013, p.31-4). Los diagramas visuales con cajas negras o signos de interrogación, por un lado, y los términos de relleno, ${ }^{12}$ por otro, son las marcas más empleadas para representar dichos vacíos.

(b) Descripción idealmente completa: es el modelo que incorpora entidades, propiedades, actividades y aspectos organizativos que son pertinentes para cualquier aspecto del fenómeno que se pretende explicar. Es el modelo más completo posible del continuo. En la práctica no hay modelos mecanísticos de este tipo, ya que su carácter ideal haría que tuvieran que incluir tal cantidad de factores 
potenciales que éstos resultarían casi intratables para propósitos de predicción y de control. ${ }^{13}$ Para evitar esto, en el modelo se dejan de lado detalles considerados irrelevantes para el objetivo al caso, lo cual nos permite modificar la aplicación de 'idealidad': un modelo idealmente completo será aquel que incorpore toda la información pertinente, teniendo en cuenta que ésta depende de un contexto que a su vez es un hecho objetivo del mundo que el investigador estudia.

(c) Esquemas (a lo largo del continuo): entre los dos polos se sitúan los esquemas de mecanismo que se abstraen en mayor o menor grado de los detalles más específicos de éste. Lo que cuente como grado apropiado de abstracción dependerá nuevamente del uso que pretendamos dar al modelo. Los modelos fenoménicos se asientan en el límite más alejado (alto) del esquematismo; habitualmente tienen carácter de caja negra porque no revelan nada sobre los mecanismos subyacentes. A medida que nos aproximamos al nivel más bajo de esquematismo, también lo hacemos, dicho en términos cualitativos, a la explicación EBM.

(iii) Explicar un fenómeno o el sistema que lo representa no consiste solamente en describir el comportamiento de algún mecanismo que le subyazca. También es necesario que los componentes y aspectos descritos en el modelo se correspondan de algún modo con componentes y aspectos en el mecanismo. Ahí es donde radica la plausibilidad mecanística del modelo en cuestión.

Los modelos posibles, a diferencia de los meramente fenoménicos, se proponen con el propósito de explicar. Pero su carácter es el de una conjetura débilmente constreñida en el mecanismo que da lugar al fenómeno que queremos explicar (Craver y Darden 2013, p.30-2). Son modelos que describen cómo se podrían organizar ciertas partes y actividades del fenómeno en cuestión de modo que exhibieran éste; son muy útiles heurísticamente para la construcción de un espacio de mecanismos posibles, pero no constituyen explicaciones adecuadas per sé. Craver (2006, p.361) exige no sólo que la descripción que el modelo hace sea verdadera, sino que ha de caracterizar correctamente los detalles del mecanismo.

Los modelos reales, por su parte, sí describen componentes, actividades y aspectos organizativos, todos ellos reales, del mecanismo. Y todos ellos producen de hecho el fenómeno que buscamos explicar. Son modelos que muestran el modo de funcionamiento del mecanismo, no sólo el potencial. A modo de puente entre ambos tipos de modelos, Craver sitúa los modelos plausibles. Éstos son bastante consistentes con las constricciones ya conocidas sobre los componentes, sus actividades y su organización. El grado de exactitud de la representación de los detalles del mecanismo por parte del modelo dependerá de los propósitos para los cuales se despliega el modelo. 


\section{Explicación química basada en mecanismos}

La necesidad nómica en química es controvertida (Fisher 2003, p.17). Aunque haya excepciones a la ley periódica, se puede afirmar que la Tabla Periódica es legaliforme (Scerri y McIntyre 1997) porque las leyes químicas no necesitan ser exactas para ser precisamente legaliformes (Christie 1994). En el modelo ND esta ausencia de necesidad nómica sería fatal y no podríamos hablar de explicaciones propiamente químicas. Pero dadas las carencias mencionadas del modelo ND, la ausencia de necesidad nómica no es criterio para distinguir la naturaleza de la explicación química y la de la física, desestimando o debilitando la primera. Afirmamos esto porque las leyes fundamentales de la física tampoco son rigurosamente exactas en dos sentidos: primero, porque están sometidas a condiciones ceteris paribus y, segundo, porque la 'verdad' o la 'rigurosidad descriptiva' es algo que en realidad queda reservado para las leyes fenoménicas. Esto es lo que motivó el rechazo del modelo ND por parte de Cartwright (1983).

La síntesis química es una actividad particular de la química orgánica. Veamos dos rasgos característicos de este ámbito vinculados a la crítica al modelo ND: por un lado, la explicación y la predicción no son simétricas. ${ }^{14}$ En química orgánica, explicar un fenómeno no implica que se haya podido predecir. La existencia de pocas leyes cuantitativas en química orgánica, si es que hay alguna, hace que la química orgánica teórica permita comprender fenómenos sin recurrir a leyes generales o fundamentales. ${ }^{15}$ Esto significa que, en lo que se refiere a supuestas aplicaciones de las leyes a casos complejos y concretos, la química orgánica habitualmente no explica (ni predice) los fenómenos ni los problemas de aproximación por medio de leyes matemáticas que 'cubran' dichos casos, al modo del modelo ND. ${ }^{16}$ Consideramos que una manera de responder mejor a esta forma de generar conocimiento y explicaciones es mediante el recurso a mecanismos que sustentan explicaciones.

Por otro lado, podemos identificar los aspectos de un mecanismo (Darden 2006, p.279-80) en la síntesis química del siguiente modo. El componente o entidad es la estructura de una molécula orgánica. Puede ser un compuesto complejo que se dé en la naturaleza (esteroides, vitaminas) y también alguna estructura que desafíe tanto al conocimiento teórico actual como al alcance de las tecnologías de diseño sintético, dado su componente de producto que hay que crear. El objetivo de la síntesis química es encontrar la manera de crear la molécula que nos interesa por medio de técnicas propias de la química orgánica.

La organización 'mecanística' es una secuencia de reacciones químicas que se elige cuidadosamente en un orden rígido (Corey 1969, p.179) y que debe satisfacer tres requisitos que den cuenta, aunque no necesariamente, de los aspectos temporales y de las localizaciones contextuales: (i) los pasos de la secuencia deben contar con condiciones bien especificadas de reacción; (ii) los reactivos o bien ya deben estar en

Principia 18(3): 393-417 (2014). 
disposición de empleo para los químicos, o bien ya son producto de algún paso previo; y (iii) cada reacción debe producir un producto que deseamos y cuya estructura está especificada, de manera que nos suministre reactivo suficiente para siguientes pasos-reacciones. Junto con ello, debemos tener en cuenta que la cantidad de modelos posibles para un mismo problema de síntesis química (fenómeno) puede ser múltiple y que la elección de uno de ellos puede requerir criterios de plausibilidad práctica, económica e incluso estética.

La propuesta mecanística de explicar un mecanismo de reacción a través de una secuencia de reacciones intermedias es algo que ya Morrison (1999) destacaba cuando se refería a los 'modelos mediadores' (cfr. Morrison 1999, p.10-37; Fisher 2003, p.19). Algo análogo les sucede a las cadenas de mecanismos sobre los que se fundamentan muchas explicaciones basadas en mecanismos de reacción.

\subsection{Estrategias en Síntesis Química}

La primera tarea en síntesis química consiste en la elaboración de mecanismos de síntesis. Éstos no son sino las secuencias de pasos o etapas conducentes al objetivo que se pretende sintetizar. Cuando el plan sintético se diseña, el químico emplea algún tipo de reacción química ya caracterizado exitosamente por la química orgánica en términos estructurales de reactivos y productos. Un ejemplo paradigmático es la reacción Diels-Alder (Fig. 2):

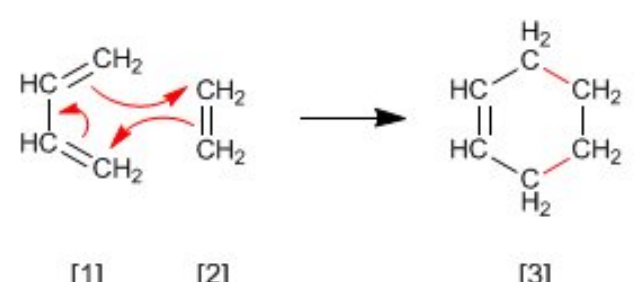

Figura 2: Reacción Diels-Alder

El alqueno [2] y el dieno conjugado [1] producen un anillo de seis miembros con un enlace doble [3]. El empleo de esta reacción en un plan sintético permite generar un anillo ciclohexeno no presente previamente (Corey y Chelg 1995, p.6-8). Varias transformaciones de este tipo encadenadas permiten generar una secuencia que parte de reactivos químicos ya disponibles (como [1] y [2]), que avanza por pasos estructuralmente especificados que producen los productos deseados y que finaliza con la molécula sintética que se pretende obtener.

El modelo plausible que el experimentador emplee aquí hará que éste se enfrente a una nueva situación que podemos caracterizar según dos ejes básicos: por un 
lado, tendrá que elegir de hecho un rango limitado de ejemplares de reactivos y de reacciones, así como las condiciones de éstas para los pasos individuales (componentes, ordenación y contexto mecanísticos). Por otro lado, deberá ejecutar, analizar y optimizar los experimentos apropiados (proceso dinámico temporal del mecanismo) (Burewicz y Miranowicz 2003, p.245; Corey y Chelg 1995, p.2). La fase de ejecución exige conocimiento obtenido a partir de resultados experimentales, amén de "habilidades" de laboratorio y otros elementos extra-teóricos. ${ }^{17}$

Para vincular directamente el plan del diseño y el conocimiento específicamente químico-orgánico, una EBM exige que, para conectar exitosamente varias series de reacciones que nos conduzcan a obtener una molécula que buscamos, hemos de ser capaces de identificar (1) los compuestos iniciales potenciales y plausibles, y (2) alguna secuencia de reacciones bien caracterizadas estructuralmente (Carey y Sundberg 1984, p.194). El fin del uso de ambos elementos es obtener la molécula pretendida. Para obtener los compuestos iniciales potenciales y plausibles, hemos de reconocerlos entre varios ítems alternativos; para llevar (2) a buen puerto, hemos de producir e interpretar las fórmulas estructurales y las reacciones químicas. Éste es el trasfondo 'mecanístico' del conocimiento empírico químico y de las habilidades que dan forma a la caja de herramientas en el ámbito del diseño sintético.

El diseño de la síntesis de una molécula compleja es por lo tanto un reto en el sentido de que es una hipótesis abductiva, una inferencia de la mejor explicación. De modo contrafáctico, el experimentador tiene que extrapolar su conocimiento sobre las reacciones orgánicas a un nuevo territorio con el fin de resolver un problema práctico. Los químicos no tienen experiencia sobre cómo esa molécula pretendida particular responderá a los intentos de ejecutar reacciones químicas específicas, pero deberán ser capaces de dirigir la secuencia de reacciones que dé como resultado dicha molécula. Su herramienta será el conocimiento sobre cómo responden las moléculas más simples en reacciones químicas semejantes. Esto les servirá para estimar la efectividad potencial de las reacciones o secuencias de reacciones que podrían resultar parte de un plan sintético.

\subsection{EBMs, Intervenciones y Dinamismo de las Reacciones Químicas}

Los químicos reconocen que una ecuación química global equilibrada no expresa mucho acerca de cómo se llevó a cabo la reacción. Es decir, reconocen que no explica (Ramsey 2008, p.974). A lo sumo, tan sólo describe la suma de una serie de reacciones sencillas (etapas elementales o reacciones elementales) que representan o describen el avance de la reacción global en un nivel molecular. Toda la secuencia de pasos elementales que conduce a la formación del producto es el mecanismo de reacción. Lo que nos interesa aquí es mostrar claramente la característica explicativa de un mecanismo de reacción química.

Principia 18(3): 393-417 (2014). 
Tengamos en cuenta que las dudas previas sobre el carácter explicativo de un mecanismo apuntan al hecho de que básicamente no hay un formato universal para representar los mecanismos de reacción (Friesen 2008, p.1518). Los mecanismos son una forma importante de explicar por qué las reacciones conducen a la obtención de los productos que proporcionan, por qué lo hacen a cierta velocidad y por qué en condiciones contrafácticas los productos obtenidos son otros. Frente a otras propuestas (Carpenter 2000; Goodwin 2003), el enfoque EBM (Machamer et al. 2000; Craver y Darden 2005, 2013) tiene aún que mostrar si es igualmente útil o no a la hora de hacer explícito el uso explicativo de los mecanismos por parte de los químicos, entre ellos los orgánicos.

Sostiene Ramsey (2008, p.971), en la línea de Tabery (2004, p.10), que un mecanismo en química orgánica es EBM (explicativo) cuando da cuenta del proceso dinámico que genera un cambio desde los reactivos a los productos. Enfatiza el carácter causal, intervencionista y contingente (no basado en leyes estrictas) de este tipo de explicación porque, primero, los mecanismos se construyen mediante intervenciones y, segundo, porque los mecanismos sólo tienen éxito cuando muestran cómo algunas hipótesis dependen de otras (Woodward 2003, p.375). Es decir, hacen un seguimiento desde unas contingencias a otras, pero sin intentar disminuir su grado contingente (no necesario) al procurar dar lugar a explicaciones. ${ }^{18}$

Esta manera de enfocar las EBMs es interesante, pero no tan novedosa cuando parece indicar que el enfoque clásico de Machamer, Darden y Craver (2000), y otros posteriormente, es insuficiente porque 'supuestamente' se limita a ser un mero listado de entidades, actividades y de una organización espacial y temporal (Tabery 2004, p.10-1; Ramsey 2008, p.274). Lo que falta en el esquema explicativo EBM es el factor de 'producción dinámica' de las reacciones, según Tabery (2004, p.12). Para Tabery (2004) y Ramsey (2008), un mecanismo de reacción no se limita a especificar únicamente transformaciones de estado o únicamente actividades, sino que además da cuenta de cómo se genera una transformación de estado y dice que las actividades son ítems productivos situados en redes interactivas. Esta productividad se especifica en cambios particulares de propiedad en un mecanismo particular (Tabery 2004, p.11). Esta especificación es explicativa porque dice cómo pueden producirse los cambios de propiedad y porque reafirma que estos efectos son de hecho tales cambios.

Una consecuencia aparente de las afirmaciones de Ramsey (2008, p.974-5) es que es el análisis de Tabery — que recurre a la concepción de Ingold (1953)— el que da sentido a la práctica explicativa en química (orgánica), no el análisis de la concepción clásica de EBM (Machamer, Darden, Craver 2000). Según la tesis de RamseyTabery, esta concepción no ha dado sentido a la práctica explicativa química hasta la fecha en parte porque se ha concentrado en el análisis de la explicación en ciencias de la vida y en neurociencias (Tabery 2004, p.975) y, según ellos, es un tipo de explicación mecanística distinto al de la química. Lo que a nosotros nos interesa es 
incorporar a dicha concepción el factor de producción dinámica que creemos conveniente identificar en cualquier mecanismo de reacción química, amén de hacer ver que la propuesta de modelos posibles y, ante todo, de los modelos plausibles de un mecanismo, es un elemento consustancial a la explicación de una reacción.

El carácter propiamente explicativo de un mecanismo niega por lo general que éste sea deductivo. Aunque en el curso de la explicación de una reacción se hagan muchas deducciones, la explicación global no es deductiva. Puede haber mecanismos más o menos generales, pero no hay leyes generales sobre los comportamientos de los sistemas reactivos. El carácter dinámico que las EBM incorporan hace más interesante reconocer su naturaleza causal e intervencionista en el caso de las reacciones químicas (orgánica) (cfr. Psillos 2002, p.81-133). ${ }^{19}$ Una EBM exitosa mostrará, como ya dijimos, patrones de dependencia contrafáctica que describen cómo un sistema cuyo comportamiento deseamos explicar cambiaría en condiciones varias (Woodward 2002, p.96). En este tipo de explicaciones se forman generalizaciones y éstas son invariantes en un sentido: apoyan contrafácticos interventores. Es decir: contrafácticos que se mantendrán incluso cuando ciertos valores de determinadas variables hayan cambiado.

La intervención también es una característica que nos interesa rescatar para nuestro objetivo de aplicar el enfoque EBM a la química orgánica porque, aun contando con la concepción clásica de Machamer, Darden y Craver (2000) y otros (Glennan 1996), ésta no es capaz de suministrar todos las ocurrencias causales detalladas de una reacción o de una serie de reacciones químicas. Las ocurrencias serán modeladas como posibles en primer lugar, como plausibles posteriormente y, finalmente, como reales. Con el cuerpo de modelos plausibles de un mecanismo a mano, el químico construirá alguna generalización mediante intervenciones. Pretenderá ver qué series de generalizaciones contrafácticas se pueden apoyar cuando intervenimos en el sistema estudiado.

\subsection{Inferencia de Mecanismos para una Explicación}

Las explicaciones construidas sobre la base de intervenciones son contingentes en el sentido de los dos elementos de partida arriba mencionados: su dependencia reticular teórica y su recurso a la disposicionalidad. Esto toma cuerpo en diversos aspectos. Mencionemos tres: (A) las evidencias para una EBM son a menudo indirectas, (B) las condiciones en que se desarrolla la EBM son un elemento fundamental, y (C) pueden existir varias EBMs válidas acerca de un mismo fenómeno que compitan entre sí.

Para poder llegar a obtener una EBM exitosa, el químico parte de datos de experimentación a partir de los cuales, entre otros elementos, se infiere la EBM. Los datos iniciales suelen ser datos sobre la velocidad de la reacción en diferentes solventes, a diferentes temperaturas y en diferentes concentraciones de los reactivos (cinética 
de reacción). La velocidad es el cambio en la concentración de reactivos o productos respecto al tiempo. En una reacción 'reactivos $\rightarrow$ productos' se expresa que, en el curso de ésta, las moléculas de reactivos se consumen mientras se forman moléculas de productos. Se puede seguir el progreso de la reacción (el mecanismo) mediante la monitorización de la disminución en la concentración de reactivos o del incremento en la de los productos. En el caso de la reacción de bromo molecular con ácido fórmico, la disolución es acuosa y el bromo molecular reacciona con el ácido fórmico $(\mathrm{HCOOH})$ del siguiente modo:

$$
\mathrm{Br}_{2}(\mathrm{ac})+\mathrm{HCOOH}(\mathrm{ac}) \rightarrow 2 \mathrm{Br}^{-}(\mathrm{ac})+2 \mathrm{H}^{+}(\mathrm{ac})+\mathrm{CO}_{2}(g)^{20}
$$

El color del bromo molecular es rojizo-café y, mientras la reacción avanza, la concentración de $\mathrm{Br}_{2}$ disminuye con rapidez. Este cambio se puede monitorizar si medimos el desvanecimiento del color del bromo molecular con un espectrofotómetro o aparato de medición de espectros luminosos (Chang 1998, p.509-10). El resultado de la monitorización suele ser una ley de velocidad, en cuyos casos límite se observa una ley de segundo orden (velocidad $=k_{2}[A][B]$ ), en la que la velocidad es proporcional a dos especies, o una ley de primer orden (velocidad $=k_{1}[A]$ ), en la que lo es sólo a una especie. Es importante destacar que la ley de velocidad se determina experimentalmente (Chang 1998, p.515). Para ello, lo primero que se hace es medir la velocidad. Si la reacción es en disolución, la concentración de muchas especies se puede monitorizar por métodos espectrofotométricos; si hay iones involucrados, la monitorización se puede realizar mediante mediciones de la conductancia eléctrica, y en el caso de reacciones que involucran gases, la monitorización se lleva a cabo midiendo la presión (Cfr. Chang 1998, p.509).

Pues bien, a partir de las dependencias descubiertas en las leyes de velocidad, los químicos infieren el mecanismo que produciría la ley de velocidad observada experimentalmente. Para ello recurren a un conjunto de etapas elementales (que Ingold estableció por primera vez en 1953). Por ejemplo, en la reacción entre óxido nítrico y oxígeno (Chang 1998, p.533),

$$
2 \mathrm{NO}(g)+\mathrm{O}_{2}(g) \rightarrow 2 \mathrm{NO}_{2}(g)
$$

como dijimos anteriormente, se sabe experimentalmente que el producto no se forma directamente como resultado de la colisión de una molécula de $\mathrm{NO}$ con una de $\mathrm{O}_{2}$ (aspecto (A)). Y se sabe porque la especie $\mathrm{N}_{2} \mathrm{O}_{2}$ se ha detectado experimentalmente en el transcurso de la reacción. Pues bien, se propone entonces un modelo posible de mecanismo si suponemos que la reacción se realiza en dos etapas elementales como las siguientes:

$$
2 \mathrm{NO}(g) \rightarrow \mathrm{N}_{2} \mathrm{O}_{2}(g)
$$


(2 $\stackrel{\text { a }}{ }) \mathrm{N}_{2} \mathrm{O}_{2}(g)+\mathrm{O}_{2}(g) \rightarrow 2 \mathrm{NO}_{2}(g)$

En la etapa $1^{\mathrm{a}}$, dos moléculas de NO chocan entre sí para formar una molécula de $\mathrm{N}_{2} \mathrm{O}_{2}$. En la etapa $2^{-}$, se forman dos moléculas de $\mathrm{NO}_{2}$. La ecuación global química, que representa el cambio total, está dada por la suma de las dos etapas (Chang 1998, p.533-5):

\begin{tabular}{|c|c|c|c|}
\hline $\mathrm{NO}+\mathrm{NO}$ & $\rightarrow$ & $\mathrm{N}_{2} \mathrm{O}_{2}$ & (Etapa $1^{\mathrm{a}}$ ) \\
\hline $\mathrm{N}_{2} \mathrm{O}_{2}+\mathrm{O}_{2}$ & $\rightarrow$ & $2 \mathrm{NO}_{2}$ & (Etapa $2^{\mathrm{a}}$ ) \\
\hline $2 \mathrm{NO}+\mathrm{N}_{2} \mathrm{O}_{2}+\mathrm{O}_{2}$ & $\rightarrow$ & $\mathrm{N}_{2} \mathrm{O}_{2}+2 \mathrm{NO}_{2}$ & (Reaccic \\
\hline
\end{tabular}

Aquí $\mathrm{N}_{2} \mathrm{O}_{2}$ son las especies intermediarias, pues aparecen en el mecanismo de la reacción (en las etapas), si bien no en la ecuación global equilibrada. Conocer las etapas de reacción permite deducir la ley de velocidad, pero en general los químicos reconocen que no se puede decir a simple vista, a partir de la ecuación equilibrada global, si la reacción ocurre tal como se muestra o si ocurre en etapas.

Veamos un caso de elucidación de mecanismo de reacción mediante estudios experimentales: la descomposición del peróxido de hidrógeno (agua oxigenada: $\mathrm{H}_{2} \mathrm{O}_{2}$ ) . La reacción global es la siguiente:

$$
2 \mathrm{H}_{2} \mathrm{O}_{2}(a c) \rightarrow 2 \mathrm{H}_{2} \mathrm{O}(l)+\mathrm{O}_{2}(g)
$$

Los resultados experimentales indican que la ley de velocidad es 'Velocidad = $k\left[\mathrm{H}_{2} \mathrm{O}_{2}\right]\left[\mathrm{I}^{-}\right]$' (el ion $\mathrm{I}^{-}$aparece y desaparece en etapas intermedias del mecanismo, etapas representadas por modelos distintos de mecanismos). La reacción es de primer orden respecto a ambos elementos: $\mathrm{H}_{2} \mathrm{O}_{2} \mathrm{y} \mathrm{I}^{-}$. La descomposición no ocurre en una sola etapa que se vea reflejada en la ecuación global equilibrada, pues si así fuera, la reacción sería de segundo orden respecto a $\mathrm{H}_{2} \mathrm{O}_{2}$ (como resultado de la colisión de dos moléculas de $\mathrm{H}_{2} \mathrm{O}_{2}$ ). Y el ion $\mathrm{I}^{-}$, que no aparece en la ecuación global, sí aparece en la ley de velocidad. Pues bien, ¿cómo se explica todo esto?

Para explicar la ley de velocidad, se propone un modelo de mecanismo en el que la reacción se realiza en dos etapas elementales, cada una bimolecular:

$$
E_{1}: \mathrm{H}_{2} \mathrm{O}_{2}+\mathrm{I}^{-} \underset{k_{2}}{\rightarrow} \mathrm{H}_{2} \mathrm{O}+\mathrm{OI}^{-} \quad E_{2}: \mathrm{H}_{2} \mathrm{O}_{2}+\mathrm{I}^{-} \underset{k_{1}}{\rightarrow} \mathrm{H}_{2} \mathrm{O}+\mathrm{O}_{2}+\mathrm{I}^{-}
$$

Si se supone que $E_{2}$ es mucho más rápida que $E_{1}$, entonces la velocidad global resulta controlada por la velocidad de $E_{1}$, conocida como velocidad limitante de la reacción. El paso limitante de la reacción es la etapa o paso más lento en la secuencia que conduce a la formación de los productos. En este caso, tras formarse el ion $\mathrm{OI}^{-}$ en $E_{1}$, se consume inmediatamente en $E_{2}$; por ello, la velocidad de reacción se puede determinar sólo a partir de $E_{1}$ : 'Velocidad $=k_{1}\left[\mathrm{H}_{2} \mathrm{O}_{2}\right]\left[\mathrm{I}^{-}\right]$'. Hay que fijarse en que 
$\mathrm{OI}^{-}$es un intermediario, pues no aparece en la ecuación global equilibrada, como tampoco lo hace el ion $\mathrm{I}^{-}$, que se distingue de $\mathrm{OI}^{-}$en que $\mathrm{I}^{-}$está presente al principio de la reacción y cuando se ha completado. La función de $\mathrm{I}^{-}$es acelerar la reacción. Por ello se denomina 'catalizador'.

Sin embargo, la cinética de una reacción no es un indicador siempre completo del mecanismo. En química, gran parte del trabajo se dedica a distinguir entre mecanismos auténticos y meros modelos fenoménicos suministrados por los estudios cinéticos. La evidencia cinética puede que no proporcione las verdaderas dependencias de velocidad. No hay por qué esperar una correlación unívoca uniforme entre mecanismo y orden de reacción. Para juzgar el papel del orden de reacción, hay que tener en cuenta todas las circunstancias (Ingold 1953, p.314; Ramsey 2008, p.978). Las evidencias experimentales - las cinéticas y las de otros tipos- permiten inferir modelos posibles de un curso de reacción, y si más de uno es adecuado, el químico los trata como modelos plausibles del mecanismo. Lo que habitualmente no encuentra el químico es un mecanismo general para un tipo de reacción dada. Si contrafácticamente variamos las condiciones, puede que los mismos reactivos den lugar a productos diferentes. E incluso en las mismas condiciones, más de un modelo plausible puede ser válido como mecanismo del mismo fenómeno (aspecto (C)) (Grossman 2003, p.51-6; Groutas 2000, p.65-8). Esto sucede de este modo básicamente porque los químicos ofrecen mecanismos contingentes construidos a partir de intervenciones, los cuales sirven de fundamento para que podamos inferir que las explicaciones químicas se basan en ellos.

A modo de resumen, por lo tanto, el análisis de un mecanismo de reacción química que sirva de base para la explicación, amén del factor dinámico, debe considerar lo siguiente: (i) la recolección de datos (mediciones de velocidad), (ii) el análisis de dichos datos para determinar la constante de velocidad y el orden de reacción, a partir de lo cual se escribe la ley de velocidad; y (iii) la inferencia de uno o más modelos plausibles del mecanismo para la reacción, en términos de etapas elementales. Éstas deben satisfacer dos requisitos: (1) la suma de las etapas elementales debe proporcionar la ecuación global equilibrada para la reacción, y (2) la etapa determinante de la reacción debe predecir la misma ley de velocidad que la ley determinada experimentalmente.

\section{Conclusiones}

La función reflexiva, pasiva, basada en la observación, que la filosofía asignaba en la estela del empirismo lógico a la ciencia ha sido sustituida por una concepción más próxima a la actividad científica, que reconoce el papel predominante del hacer en ella. Entre las prácticas científicas se encuentran las prácticas de indagación explica-

Principia 18(3): 393-417 (2014). 
tiva, que pretenden explicar fenómenos que ocurren en la naturaleza o que se crean en el laboratorio, más allá de su mero diseño y descripción. Esta perspectiva, que no desestima el posible recurso de los químicos a leyes fundamentales putativas de la física, nos permite observar que la química orgánica habitualmente no se fundamenta en ellas. Más allá de la ley periódica, no parece justificado calificar los principios de la química, incluso sus 'leyes' fenoménicas, como leyes fundamentales (cfr. Cartwright 1983, p.54-7).

Al igual que ocurre en las ciencias de la vida y en las neurociencias, la noción de explicación desempeña un papel considerablemente selecto en química, aunque mantiene sus propias particularidades. En vez de tomar la forma tradicional de la explicación ND, dependiente de leyes teóricas no fenomenológicas, las explicaciones que se emplean mayoritariamente en química se pueden conceptualizar en términos de un enfoque EBM complementado con elementos que enfatizan el aspecto dinámico de los fenómenos de reacción química. Para hacer esto explícito, hemos propuesto la explicación mecanística (o basada en mecanismos) como marco filosófico para nuestro análisis de las prácticas de indagación explicativa en química orgánica. Este marco ha sido criticado desde la filosofía de la química (Tabery, 2004; Ramsey, 2008) debido a lo que aparentemente no es sino un enfoque excesivamente estático de los procesos de modelación de los mecanismos de fenómenos de reacción. Ante esto, nuestra alternativa ha consistido en analizar varios procedimientos de examen químico de producción de fenómenos. El resultado indica que en química orgánica, sus prácticas se dirigen a proponer modelos (posibles, plausibles y reales) de mecanismos que den cuenta del proceder diacrónico de las reacciones químicas orgánicas. Se trata de prácticas con un carácter intervencionista visible en las variaciones realizadas en las condiciones de reacción o en los propios reactivos, bajo un patrón contrafáctico, que permiten derivar la pertinencia de unos mecanismos y la irrelevancia de otros. La obtención de estos mecanismos es lo que posteriormente permite inferir una explicación de los datos experimentales y de las ecuaciones globales equilibradas obtenidas previamente de un modo empírico.

Dado que en la química, en particular en la orgánica, los aspectos dinámicos y diacrónicos de las reacciones son indispensables si queremos mostrar cómo explican ciertos fenómenos, hemos propuesto un esbozo de refinamiento de la noción clásica de EBM al añadir los conceptos de dinamismo, intervención y modalidad (contrafácticos). Esto se observa en los ejemplos de análisis en química sintética y en los procedimientos mecanísticos de las reacciones químicas orgánicas, en los cuales el factor dinámico es una de las características más notables para justificar por qué un mecanismo sirve de base para una explicación. Esto, por supuesto, encaja con el punto de partida en el que enfatizábamos la importancia de las prácticas y del carácter experimental del conocimiento, dado que el grado de intervención en química orgánica y sintética es imprescindible a la hora de inferir modelos plausibles de mecanismos

Principia 18(3): 393-417 (2014). 
que ayuden a explicar las reacciones estudiadas. Esta tarea intervencionista se puede concebir en términos de estrategias contrafácticas utilizadas para obtener en último término los mecanismos reales que definitivamente sean el fundamento de la explicación de una reacción.

\section{Agradecimientos}

Trabajo elaborado con el apoyo de la Secretaría de Estado de Investigación, Desarrollo e Innovación del Ministerio de Economía y Competitividad de España (Proyecto de Investigación con referencia FFI2013-42154-P, 'La evaluación de beneficios como ciencia reguladora: las declaraciones de salud de los alimentos funcionales'), cofinanciado con fondos FEDER de la Comisión Europea.

\section{References}

Balzer, W.; Moulines, C. U. (eds.). 1996. Structuralist Theory of Science: Focal Issues, New Results. Berlin: de Gruyter.

Beatty, J. 1995. The Evolutionary Contingency Thesis. In: G. J. Wolters; J. G. Lennox (eds.) Theories and Rationality in the Biological Sciences: The Second Annual Pittsburgh/Konstanz Colloquium in the Philosophy of Science. Pittsburgh: University of Pittsburgh Press, p.4581.

Bechtel, W. 2007. Biological Mechanisms: Organized to Maintain Autonomy. In: F. C. Boogerd; F. J. Bruggeman; J.-H. S. Hofmeyr; H. V. Westerhoff (eds.) Systems Biology: Philosophical Foundations. New York: Elsevier, p.269-302.

2011. Mechanism and Biological Explanation. Philosophy of Science 78: 533-57.

Bechtel, W; Richardson, R. C. 2010. Discovering Complexity: Decomposition and Localization as Strategies in Scientific Research. Second Edition. Cambridge, MA: MIT Press/Bradford Books (original de 1993).

Bengoetxea, J. B.; Todt, O.; Luján, J. L. 2013. El concepto de información mecanística en la evaluación de riesgos. Contrastes, Suplemento 18: 13-26.

-2014. Similarity and representation in chemical knowledge practices. Foundations of Chemistry 16(3): 215-33.

Berson, J. A. 2008. Fundamental theories and their empirical patches. Foundations of Chemistry 10: 147-56.

Bogaard, P. A. 1981. The Limitations of Physics as a Chemical Reducing Agent. Philosophy of Science Association 1978(2): 345-36.

Buchwald, J. Z. 1994. The Creation of Scientific Effects: Heinrich Hertz and Electric Waves. Chicago: The University of Chicago Press.

Burewicz, A.; Miranowicz, N. 2003. Beyond the Dimensionality of Visualization in Chemistry. In: J. E. Earley, Sr. (ed.) Chemical Explanation: Characteristics, Development, Autonomy. New York: New York Academy of Sciences, p.244-9.

Carey, F. A.; Sundberg, R. J. 1984. Advanced Organic Chemistry. Part A: Structure and Mechanisms. Second Edition. New York: Springer Science. 
Carpenter, B. 2000. Models and Explanations: Understanding Chemical Reaction Mechanisms. In: N. Bhushan; S. Rosenfeld (eds.) Of Minds and Molecules: New Philosophical Perspectives on Chemistry. New York: Oxford University Press, p.211-29.

Carroll, F. A. 1998. Perspectives on Structure and Mechanism in Organic Chemistry. Pacific Grove, CA: Brooks/Cole.

Cartwright, N. 1983. How the Laws of Physics Lie. Oxford: Clarendon Press.

Chamizo, J. A. 2013. About Chemical Experiment. In: J.-P. Llored (ed.) The Philosophy of Chemistry: Practices, Methodologies, and Concepts. Newcastle upon Tyne: Cambridge Scholars, p.628-39.

Chang, H. 2004. Inventing Temperature: Measurement and Scientific Progress. Oxford: Oxford University Press.

Chang, R. 1998. Chemistry, Sixth Edition. Nueva York: McGraw-Hill.

. 2012. Is Water H2O? Evidence, Realism and Pluralism. Dordrecht: Springer.

Christie, M. 1994. Philosophers versus Chemists Concerning 'Laws of Nature'. Studies in History and Philosophy of Science 25: 613-29.

Corey, E. J. 1969. Computer-Assisted Design of Complex Organic Syntheses. Science 166 (3902): 178-92.

Corey, E. J.; Chelg, X.-M. 1995. The Logic of Chemical Synthesis. New York: John Miley \& Sons.

Craver, C. F. 2002. Interlevel Experiments, Multilevel Mechanisms in the Neuroscience of Memory. Philosophy of Science, Supplement 69: S83-S97.

- 2005. Beyond Reduction: Mechanisms, Multifield Integration and the Unity of Neuroscience. Studies in History and Philosophy of the Biological and Biomedical Sciences 36: 373-95.

- 2006. When Mechanistic Models Explain. Synthese 153: 355-76.

- 2007. Explaining the Brain: Mechanisms and the Mosaic Unity of Neuroscience. Oxford: Clarendon Press.

- 2013. Functions and Mechanisms: A Perspectivalist View. In: P. Huneman (ed.) Functions: Selection and Mechanisms. Dordrecht: Springer, p.133-58.

Craver, C. F.; Bechtel, W. 2006. Mechanism. In: S. Sarkar; J. Pfeifer (eds.) The Philosophy of Science: An Encyclopedia. New York: Routledge, p.469-478.

Craver, C. F; Darden, L. 2001. Discovering Mechanisms in Neurobiology: The Case of Spatial Memory. In: P. Machamer; R. Grush; P. Mclaughlin (eds.) Theory and Methods in the Neurosciences. Pittsburgh: University of Pittsburgh Press, p.112-37.

- 2013. In Search of Mechanisms: Discoveries across the Life Sciences. Chicago: The University of Chicago Press.

Darden, L. 2006. Reasoning in Biological Discoveries: Mechanisms, Interfield Relations, and Anomaly Resolution. New York: Cambridge University Press.

De Ridder, J. 2006. Mechanistic artefact explanation. Studies in History and Philosophy of Science 37: 81-96.

Elgin, M.; Sober, E. 2002. Cartwright on explanation and idealization. Erkenntnis 57: 441-50.

Fisher, G. 2003. Explaining Explanation in Chemistry. In: J. E. Earley, Sr. (ed.) Chemical Explanation: Characteristics, Development, Autonomy. New York: The New York Academy of Sciences, p.16-21.

Fleck, L. 1935 (1979). Genesis and Development of a Scientific Fact. Chicago: The University of Chicago Press.

Principia 18(3): 393-417 (2014). 
Forge, J. 1998. Explanation and Mechanism: Reflections on the Ontic Conception of Explanation. In: D. Anapolitanos; A. Baltas; S. Tsinorema (eds.) Philosophy and the Many Faces of Science. Lanham, MD: Rowman and Littlefield, p.76-92.

Franklin, A. 1990. Experiment, Right or Wrong. Cambridge: Cambridge University Press.

Friesen, J. B. 2008. Saying What You Mean: Teaching Mechanisms in Organic Chemistry. Journal of Chemical Education 85(11): 1515-1518.

Galison, P. 1996. Image and Logic: A Material Culture of Microphysics. Chicago: The University of Chicago Press.

Glenann, S. 1996. Mechanisms and the nature of causation. Erkenntnis 44(1): 49-71.

- 2005. Modeling Mechanisms. Studies in History and Philosophy of Biological and Biomedical Sciences 36: 443-64.

Goodwin, W. 2003. Explanation in Organic Chemistry. In: J. E. Earley, Sr. (ed.) Chemical Explanation: Characteristics, Development, Autonomy. New York: Annals of the New York Academy of Science, p.141-53.

- 2008. Structural formulas and explanation in organic chemistry. Foundations of Chemistry 10: 117-27.

Gould, S. J. 1986. Evolution and the Triumph of Homology, or Why History Matters. American Scientist 74: 60-9.

Grossman, R. B. 2003. The Art of Writing Reasonable Organic Reaction Mechanisms. Second Edition. Nueva York: Springer.

Groutas, W. C. 2000. Organic Reaction Mechanisms. New York: John Wiley \& Sons.

Hacking, I. 1983. Representing and Intervening: Introductory Topics in the Philosophy of Natural Science. Cambridge: Cambridge University Press.

- 1989. Philosophers of Experiment. In: A. Fine; J. Leplin (eds.) PSA: Proceedings of the Biennial Meeting of the Philosophy of Science Association, Vol. 2 (1988). Chicago: The University of Chicago Press-PSA, p.147-56.

- 1992. The Self-Vindication of the Laboratory Sciences. In: A. Pickering (ed.) Science as Practice and Culture. Chicago: The University of Chicago Press, p.29-64.

Harré, R. 2004. Modeling: Gateway to Nature. New York: Springer.

- 2009. Pavlov's Dogs and Schrödinger's Cat. Oxford: Oxford University Press.

Hempel, C. G. 1965. Aspects of Scientific Explanation. New York: The Free Press.

Harré, R.; Oppenheim, P. 1948. Studies in the logic of explanation. Philosophy of science 15(2): 135-175.

Hendry, R. F. 1999. Theories and Models: the Interactive View. In: M. S. Morgan; M. Morrison (eds.) Visual Representations and Interpretations. London: Springer, p.121-30.

- 2001. Mathematics, Representation and Molecular Structure. In: U. Klein (ed.) Tools and Modes of Representation in Laboratory Sciences. Dordrecht: Kluwer, p.221-36.

Hoffmann, R. 1995. The Same and Not the Same. New York: Columbia University Press. 1998. Qualitative thinking in the age of modern computational chemistry-or what Lionel Salem knows. Journal of Molecular Structure (Theochem). 424: 1-6.

Kitcher, P; Salmon, W. (eds.) 1989. Scientific Explanation. Minneapolis: University of Minnesota Press.

Liegener, C.; Del Re, G. 1987. Chemistry vs. Physics, the Reduction Myth, and the Unity of Science. Zeitschrift für allgemeine Wissenschaftstheorie 18(1-2): 165-74.

Lipton, P. 2004. Inference to the Best Explanation. Second Edition. London: Routledge.

Principia 18(3): 393-417 (2014). 
Llored, J.-P. (ed.). 2013. The Philosophy of Chemistry: Practices, Methodologies, and Concepts. Newcastle upon Tyne: Cambridge Scholars.

Machamer, P. 2004. Activities and Causation: The Metaphysics and Epistemology of Mechanisms. International Studies in the Philosophy of Science 18: 27-39.

Machamer, P.; Darden, L.; Craver, C. F. 2000. Thinking about Mechanisms. Philosophy of Science 67(1): 1-25.

Morrison, M. 1999. Models as Autonomous Agents. In: M. S. Morgan; M. Morrison (eds.) Models as Mediators: Perspectives on Natural and Social Science. Cambridge: Cambridge University Press, p.38-65.

Nagel, E. 1961. The Structure of Science: Problems in the Logic of Scientific Explanation. New York: Harcourt, Brace and World.

Nervi, M. 2010. Mechanisms, malfunctions and explanation in medicine. Biology and Philosophy 25: 215-28.

Nunes-Neto, N. F; El-Hani, C. N. 2009. O que é função? Debates na filosofia da biologia contemporânea. Scientiae Studia 7(3): 353-401.

Psillos, S. 2002. Causation and Explanation. Bucks: Acumen.

Ramsey, J. L. 2008. Mechanisms and Their Explanatory Challenges in Organic Chemistry. Philosophy of Science 75: 970-82.

Reydon, T. A. C. 2013. Symmetry and the explanation of organismal form: Commentary on Golubitsky. In: U. Gähde; S. Hartmann;J. H. Wolf (eds.) Modeling, Simulation and the Reduction of Complexity. Berlin: De Gruyter, p.43-51.

Rosenberg, A. 1994. Instrumental Biology or the Disunity of Science. Chicago: The University of Chicago Press.

Rouse, J. 2002. How Scientific Practices Matter: Reclaiming Philosophical Naturalism. Chicago: The University of Chicago Press.

Salmon, W. 1989. Four Decades of Scientific Explanation. Pittsburgh: University of Pittsburgh Press.

Scerri, E. 1991. The Electronic Configuration Model, Quantum Mechanics and Reduction. The British Journal for the Philosophy of Science 42: 309-25.

Scerri, E.; McIntyre, L. 1997. The Case for the Philosophy of Chemistry. Synthese 111: 213-32.

Schummer, J. 2004. Why do Chemists Perform Experiments? In: D. Sobczynska; P. Zeidler; E. Zielonacka-Lis (eds.) Chemistry in the Philosophical Melting Pot. Frankfurt: Peter Lang, p.395-410.

Tabery, J. G. 2004. Synthesizing Activities and Interactions in the Concept of a Mechanism. Philosophy of Science 71: 1-15.

Theobald, D. W. 1976. Some Considerations on the Philosophy of Chemistry. Chemical Society Review 5: 203-13.

Thagard, P. 2000. Coherence in Thought and Action. Cambridge, MA: The MIT Press.

Weber, M. 2005. Philosophy of Experimental Biology. Cambridge: Cambridge University Press.

Weisberg, M. 2013. Simulation and Similarity: Using Models to Understand the World. Oxford: Oxford University Press.

Woodward, J. 2002. Experimentation, Causal Inference, and Instrumental Realism. In H. Radder (ed.) The Philosophy of Scientific Experimentation. Pittsburgh, Pa: University of Pittsburgh Press, p.87-118. 
2003. Making Things Happen: A Theory of Causal Explanation. Oxford: Oxford University Press.

JuAN BAUTISTA BENGOETXEA

Universidad de las Islas Baleares

Espanha

juanbautista.bengoechea@uib.cat

OLIVER TODT

Universidad de las Islas Baleares

Espanha

oliver.todt@uib.es

Resumo. O artigo propõe uma versão melhorada e mais de acordo com as práticas químicas da noção de explicação baseada em mecanismos. A partir da necessidade de se compreender as praticas de indagação explicativa em química orgânica de uma perspectiva dinâmica na qual a intervenção e as situações experimentais contrafactuais são de especial importância, é exposto o enfoque de explicação, não o nomológico-dedutivo, mas aquele baseado em mecanismos. A falta de uma ênfase mais pronunciada nos aspectos dinâmicos explicativos de parte deste modelo fez com que vários filósofos da química duvidassem de sua validade nesta disciplina. No artigo sustentamos que a incorporação bem articulada de tais aspectos ao enfoque de mecanismos pode salvar este e dar conta adequadamente das atividades explicativas no caso do estudo das reaçõe químicas orgânicas.

Palavras-chave: Explicação; mecanismo; química orgânica; práticas experimentais.

\section{Notas}

${ }^{1}$ Cartwright (1983) y Hacking (1983) son ya textos clásicos del nuevo experimentalismo en filosofía de la ciencia. Dos visiones panorámicas de la filosofía de las prácticas son Rouse (2002) y Chang (2012), y dos especializadas en la química son Llored (2013) y Bengoetxea, Todt y Luján (2014).

${ }^{2}$ Para el caso del efecto Hall, cfr. Hacking (1983, p.225-7). Cfr. también Buchwald (1994).

${ }^{3}$ Cfr. también Harré (2013 p.586-7).

${ }^{4}$ La química ya era en 2004 la mayor empresa científica general: un tercio de los científicos del mundo estaban involucrados en ella. Para datos al respecto, cfr. Schummer (2004 p.399).

${ }^{5}$ Recuerda Bogaard (1981, p.346) que Pauling advirtió en 1939 que sólo una pequeña parte de las contribuciones de la mecánica cuántica a la química era mecánica-cuántica pura, y que sólo unos pocos casos era de interés químico directo y alcanzados por medio de una solución precisa de la ecuación de ondas de Schrödinger.

${ }^{6}$ Ya en 1987, Liegener y Del Re (1987 p.169) critican las propuestas reduccionistas (bathogeneous) porque éstas no son capaces de suministrar modelo satisfactorio completo alguno. A lo sumo, reconocen, la explicación en ciencia podría recoger cierto grado de reducción si su objetivo fuera comprender los mecanismos de los fenómenos (Liegener y Del Re

Principia 18(3): 393-417 (2014). 
1987, p.173). También Bogaard (1981) desestima las vías reduccionistas, incluso en principio (1981, p.346), en su novedoso análisis de la teoría del enlace de valencia en química cuántica.

${ }^{7}$ Como reflejo del estado de la cuestión, véanse, por ejemplo, Kitcher y Salmon (1989), y Salmon (1989).

${ }^{8}$ Cfr. Weber (2005), Bechtel (2007) o Craver y Darden (2013) para el caso de las ciencias de la vida, Elgin y Sober (2002) y Craver (2013) para el de la biología evolutiva, Craver (2005 y 2007) para las neurociencias, y Nervi (2010) para el de la medicina.

${ }^{9}$ En lo que se refiere a la carencia de apelación a leyes, véanse Beatty (1995) y Rosenberg (1994). Craver (2007, p.107) es quien enuncia explícitamente que la explicación es una descripción de mecanismos.

${ }^{10}$ Cfr. también Glennan (2005 p.445).

${ }^{11}$ Para una crítica al enfoque mecanicista por no analizar en detalle los diferentes modos de describir los mecanismos y sus comportamientos, cfr. de Ridder (2006, p.91-4).

${ }^{12}$ Por ejemplo, tal y como señala Friesen (2008, p.1515), en la actualidad en química orgánica es desaconsejable el uso del término de relleno 'R' para designar un grupo alquilo. Aunque haya sido habitual hasta hace poco que los grupos alquilo fueran a menudo intercambiables en las reacciones, sucede que presentar una reacción típica $S_{N} 2$ con un grupo R oscurece el hecho de que el grupo saliente está enlazado con un átomo de carbono, o que la naturaleza del grupo alquilo a veces puede influir en la reactividad, dependiendo de si su carbono es primario, secundario o terciario.

${ }^{13}$ Thagard (2000, p.27) lo enuncia de este modo: un problema es intratable si no hay una solución polinómico-temporal determinista para ello; es decir, si la cantidad de tiempo necesario para resolver el problema aumenta más rápidamente que la proporción polinómica cuando el problema crece en tamaño.

${ }^{14}$ La biología evolutiva es caso paradigmático de proceso temporal y proporciona un claro contraejemplo a la tesis hempeliana de la simetría, especialmente debido a que su conceptualización es generada a partir de contingencias de la historia. Esto es, podríamos decir que la biología evolutiva da cuenta de acontecimientos biológicos únicos y que ya no se pueden repetir. Tal y como señala Stephen J. Gould (1986), si 'rebobinamos' el proceso evolutivo y lo ponemos nuevamente en marcha, no hay razón para plantear la hipótesis de la repetición. Sin embargo, si Hempel estuviera en lo correcto respecto a la evolución en tanto proceso temporal, histórico, y hubiera leyes, entonces la simetría se tendría que confirmar y el proceso 'ficticiamente' reiniciado de la evolución tendría que ser el mismo e idéntico al que se ha dado de hecho. Por lo tanto, coincidimos con las apreciaciones de Reydon (2013, p.43), para quien "the role of mathematics in biology seems quite different from its role in, for example, physics and chemistry. In these latter sciences, mathematical formalisms constitute a core feature of theories and explanations. But this is not so for the principal theories of biology. For example, evolutionary theory and evolutionary explanations, which constitute the backbone of biological science, are often presented in verbal/conceptual form without using much mathematics".

${ }^{15}$ Hacking (1983, p.164) se refiere a este tipo de fenómeno con el encabezamiento de 'A multitude of experimental laws, waiting for a theory'. Menciona la 'espera' porque efectivamente hay casos en los que finalmente se obtiene una ley general que da cuenta de fenómenos previamente explicados en términos modelo-mecanísticos y de leyes fenoménicas (cfr. 
Cartwright, 1983). Sin embargo, como señalamos en el texto, esto no es habitual en química orgánica.

${ }^{16}$ Cabría objetar que el modelo inductivo-estadístico de Hempel (en el que en un razonamiento el explanans no implica el explanandum con certeza deductiva, sino con un alto grado de probabilidad) podría servir para responder al problema planteado. Sin embargo, no creemos oportuna su consideración aquí porque continúa siendo un modelo (estructural) en el que no parece posible identificar (ónticamente) los pasos internos de los procedimientos propios en los que se generan entidades intermedias o, al menos, no permite identificarlos tan bien como permite hacerlo la explicación mecanística.

${ }^{17}$ Hendry (1999, p.125; 2001, p.227-9) propugna un enfoque interactivo de las teorías y de los modelos que reconoce la importancia del lenguaje, pero sobre todo enfatiza la de los medios extra-lingüísticos a la hora de explicar y representar —ecuaciones, diagramas, analogías, modelos, etc.

${ }^{18}$ Sobre las diversas posibilidades de rutas posibles de un mecanismo y de su interrelación mutua, cfr. Groutas (2000, p.4-9) y Grossman (2003, p.122).

${ }^{19}$ Sobre los experimentos que ponen a prueba la pertinencia causal de un mecanismo, cfr. Craver y Darden (2013, p.120-5).

20 'Ac' se refiere a una solución acuosa, 'l' al estado líquido y 'g' al estado gaseoso.

Principia 18(3): 393-417 (2014). 